19 Revue d'histoire du XIXe siècle

Société d'histoire de la révolution de 1848 et des

révolutions du XIXe siècle

$1 \mid 1985$

Varia

\title{
Considérations sur la Seconde République et les beaux-arts
}

Pierre Vaisse

\section{OpenEdition \\ Journals}

Édition électronique

URL : http://journals.openedition.org/rh19/6

DOI : $10.4000 /$ rh19.6

ISSN : $1777-5329$

\section{Éditeur}

La Société de 1848

\section{Édition imprimée}

Date de publication : 1 juin 1985

ISSN : 1265-1354

\section{Référence électronique}

Pierre Vaisse, "Considérations sur la Seconde République et les beaux-arts », Revue d'histoire du XIXe siècle [En ligne], 1 | 1985, mis en ligne le 20 juin 2005, consulté le 01 mai 2019. URL : http:// journals.openedition.org/rh19/6 ; DOI : 10.4000/rh19.6

Ce document a été généré automatiquement le 1 mai 2019.

Tous droits réservés 


\title{
Considérations sur la Seconde République et les beaux-arts
}

\author{
Pierre Vaisse
}

1 Le 15 septembre 1848, la direction des Beaux-Arts passait commande à Daumier d'une peinture, Madeleine au désert, qu'il n'acheva jamais, malgré le versement immédiat d'un acompte, et celui du solde quelques mois plus tard ${ }^{1}$. En compensation, l'État, de guerre lasse, accepta en 1863 un dessin de lui, une Marche de Silène ${ }^{2}$.

2 Symptomatique du mal qu'éprouvait l'artiste à finir ses compositions peintes, l'épisode révèle également avec quelle légèreté l'administration gérait le budget des beaux-arts. Non seulement elle versa le solde avant livraison, mais elle avait passé en septembre commande d'une œuvre sur un crédit spécial de secours aux artistes, voté par l'Assemblée nationale le 17 juillet 1848 , qui devait en principe être dépensé avant la fin de l'exercice budgétaire, c'est-à-dire de l'année civile ${ }^{3}$.

3 Dans un catalogue récent de l'œuvre peinte d'Honoré Daumier, l'affaire fait l'objet du plus étrange récit ${ }^{4}$. On y apprend que le peintre Jeanron était ministre de l'Intérieur, et François Cave toujours directeur des Beaux-Arts après la révolution de février, que le tableau avait été commandé à l'artiste à la place de l'allégorie de la République, et que le sujet en aurait été refusé par une (mystérieuse) commission de l'Académie des BeauxArts. Certes, l'auteur de la notice a pu commettre quelques lapsus, mais il ne saurait être tenu seul pour responsable d'aberrations dont l'histoire de l'art français du XIX siècle offre bien d'autres exemples, surtout lorsqu'il s'agit de l'action des pouvoirs publics en ce domaine. Il a fallu, par exemple, attendre 1980 pour que fût éclaircie l'affaire du triple concours de 1830 pour la Chambre des Députés, dont circulaient jusqu'alors des versions plus ou moins fantaisistes ${ }^{5}$. Non seulement les auteurs qui en parlaient n'avaient pas consulté les archives, mais ils n'avaient même pas pris garde au résumé, pourtant exact, que Léon Rosenthal en avait donné au début du siècle dans son livre sur la peinture sous la monarchie de Juillet ${ }^{6}$.

4 A soixante-dix ans de distance, l'ouvrage de Rosenthal reste fondamental, tout comme les thèses de François Benoît sur l'art sous la Révolution et l'Empire, et de René Schneider sur 
Quatremère de Quincy, parues quelques années plus tôt ${ }^{7}$. L'histoire de l'art d'une époque pourtant relativement récente s'appuyait alors sur l'érudition la plus solide. Elle devait bientôt s'engager dans une autre voie, du moins pour la peinture et la sculpture du XIX ${ }^{\mathrm{e}}$ siècle. Pendant une cinquantaine d'années, seuls parurent dignes de retenir l'attention les grands créateurs, les pionniers de l'art moderne. Les conditions de la vie artistique n'intéressaient qu'autant qu'ils étaient concernés, d'où la méconnaissance à peu près totale dans laquelle on se trouvait d'elles, ou des erreurs de perspective encore plus fâcheuses. Du triple concours de 1830, on ne retenait que l'esquisse de Delacroix, et le concours pour la figure peinte de la République en 1848, aurait sombré dans le même oubli que les deux autres, pour la médaille et la figure sculptée, qui eurent lieu en même temps, si Daumier n'y avait pas participé ${ }^{8}$.

Depuis une vingtaine d'années, un revirement s'est produit, un retour à la recherche patiente. Longtemps négligées, les archives de l'ancienne direction des Beaux-Arts attirent en particulier un nombre croissant de chercheurs et, bien que leur exploitation soit loin d'être achevée, elles ont ${ }^{9}$ déjà fourni matière à de précieuses études. C'est ainsi que, dans son livre The Absolute Bourgeois, T. J. Clark a pu consacrer à l'action artistique de l'État sous la Seconde République un chapitre qui, reposant sur une documentation aussi solide qu'étendue, constitue non seulement la meilleure, mais aussi la première et la seule présentation d'ensemble du sujet dont nous disposions ${ }^{10}$. Vouloir le reprendre ici dans un espace plus restreint n'aurait pas de sens ; c'est pourquoi les pages qui suivent se bornent à préciser quelques points concernant les questions administratives, et à proposer quelques vues personnelles sur le problème des Salons et des associations d'artistes.

Il semblerait que l'action des pouvoirs publics dût se juger plutôt sur les œuvres acquises ou commandées, sur les tendances artistiques favorisées par ces achats et commandes, sur l'enrichissement qui en résulta pour le patrimoine. Mais nous inclinons à penser que le temps n'est pas encore venu de porter un tel jugement. T. J. Clark s'y est essayé, mais son argumentation ressemble trop à la démonstration d'une thèse. Il conclut à un échec ; or, l'échec se définit par rapport à un but. À celui-ci, aux objectifs imprécis et changeants de l'administration des Beaux-Arts, il tend à substituer l'idéal d'un art républicain dont il déplore qu'il n'ait pas vu le jour, mais dont on voit mal ce qu'il aurait dû être.

7 En matière d'acquisitions et de commandes, un jugement solide ne peut s'appuyer (cela va de soi, mais il n'en fut pas toujours ainsi) que sur des listes exhaustives, et non, comme on a trop souvent tendu à le faire, sur l'exemple de quelques noms plus ou moins réputés. Quelles conclusions tirer lorsqu'à côté de ceux de Daumier ou Millet, on lit ceux d'Apport, de Timbel ou de Matout? Les raisons, les circonstances précises de chaque mesure particulière devraient être connues, si l'on ne veut pas interpréter comme la manifestation d'un certain goût l'effet d'une recommandation politique, ou inversement. Pour les commandes, il importe aussi de pouvoir apprécier la liberté dont l'artiste a joui dans le choix et dans la conception du sujet, ainsi que le contrôle exercé par l'administration, si l'on ne veut pas créditer celle-ci d'intentions qui lui furent toujours étrangères. Ces précautions prises, reste une tâche essentielle: voir les œuvres ellesmêmes, et savoir les regarder en s'affranchissant du manichéisme réducteur qui a si longtemps dominé l'histoire de l'art du XIX siècle. L'œuvre d'un artiste tombé dans l'oubli n'est pas nécessairement une œuvre médiocre, encore moins le produit d'une esthétique attardée. Il y aurait, enfin, quelque abus à confondre l'échelle des valeurs artistiques avec l'éventail des positions politiques ou des orientations idéologiques, et à juger un tableau d'autant plus républicain que son auteur passe pour plus révolutionnaire 
en art. Mais c'est toucher là des problèmes qui soulèvent encore, on le sait, des passions incompatibles avec les exigences d'un jugement historique.

L'histoire de la Révolution de 1848 et de la Seconde République est souvent présentée comme celle d'un flux rapidement suivi d'un reflux qui s'opère par étapes. Il est tentant d'interpréter selon le même schéma d'évolution l'action artistique de l'État, d'autant plus tentant que l'assimilation entre les deux domaines a déjà été faite par les contemporains : c'est au nom des conquêtes de la démocratie que les artistes réclamèrent une nouvelle organisation des beaux-arts. Mais il serait vain de vouloir faire coïncider la chronologie des événements d'ordre artistique avec celle des mouvements politiques. Le décalage s'explique d'autant mieux que ces mêmes artistes, pour la plupart du moins, penchaient pour une République modérée, quand ils ne préféraient pas la monarchie. Il est enfin permis de se demander s'ils ne furent pas victimes d'une illusion, si l'intensité des luttes politiques pendant le XIX ${ }^{e}$ siècle n'a pas marqué les esprits avec une force telle que le vocabulaire et les catégories qui leur étaient propres en vinrent, par extrapolation, à servir pour penser des problèmes qui, sans leur être totalement étrangers, n'en étaient cependant pas, tant s'en faut, un aspect secondaire ou le reflet pur et simple.

Le 18 mars 1848, un arrêté du gouvernement provisoire faisait entrer "les musées du Louvre, du Luxembourg, de Versailles, les galeries des anciennes résidences royales et palais du gouvernement", qui dépendaient auparavant de la Liste civile, dans les attributions du Ministère de l'Intérieur, et les trois manufactures de Sèvres, des Gobelins et de Beauvais, dans celles du Ministère de l'Agriculture et du Commerce. Il précisait la première phrase d'un arrêté signé dès le 24 février par Ledru-Rollin: "Tout ce qui concerne la direction des Beaux-Arts et des Musées, autrefois dans les attributions de la Liste civile, constituera une division du Ministère de l'Intérieur" ${ }^{11}$. En réalité, ce département ministériel comprenait déjà une division des Beaux-Arts, et cela, depuis l'époque de la Révolution ${ }^{12}$. En 1804, les musées en furent distraits pour former une direction de la Maison de l'Empereur, une situation qui devait se maintenir sous les règnes suivants. L'organisation des Salons, qui eurent lieu au Louvre jusqu'en 1848, relevait de cette administration, ainsi, par voie de conséquence, que les achats d'œuvres aux artistes vivants, qui se faisaient à cette occasion. Il s'en faut cependant que le départ des compétences entre elle et la division des Beaux-Arts du Ministère de l'Intérieur ait toujours été bien défini.

On sait qu'en 1870, les Beaux-Arts furent rattachés au Ministère de l'Instruction publique, comme ils l'avaient été au Comité de l'Instruction publique en 1791, conformément à la conception qu'en avait la pensée des Lumières. En 1848, des voix s'élevèrent pour réclamer un tel rattachement; une pétition en ce sens fut adressée à l'Assemblée nationale, et reproduite dans le Corsaire du 24 mai ${ }^{13}$. Leur maintien à l'Intérieur, au lendemain de la révolution de février, tient peut-être à l'intérêt personnel que leur portait Ledru-Rollin, puis, lors de la formation d'un nouveau ministère, en mai, à la pesanteur d'un état de fait --à moins qu'on ne pense à l'attention que la police, pendant tout le siècle, a vouée aux théâtres. Louis Napoléon Bonaparte aurait eu, avant son élection à la présidence, l'idée d'un ministère des Arts et Lettres, mais elle ne connaitra une réalisation, partielle, qu'en mai $1870^{14}$.

11 Comme les musées, les manufactures relevaient, sous les régimes précédents, et relevèrent sous le Second Empire de la Maison du souverain; elles ne passèrent à l'administration des Beaux-Arts que sous la Troisième République. Leur rattachement au Commerce et à l'Agriculture, en 1848, s'explique par l'illusion, largement répandue à 
l'époque, qu'elles jouaient ou pouvaient jouer un rôle comme modèles pour l'industrie privée ${ }^{15}$.

Aux manufactures près, le nouveau régime organisait donc, par la réunion des musées à la division déjà existante au ministère de l'Intérieur, une administration unifiée des BeauxArts. La direction des Musées, débarrassée du soin, ou du souci d'organiser les Salons annuels, conserva cependant, semble-t-il, une forte autonomie, moins peut-être par le poids de la tradition que par la personnalité du directeur, le peintre Jeanron ${ }^{16} C^{\prime}$ 'était un républicain de vieille souche, un habitué des sociétés secrètes, qui fut porté à ce poste grâce à ses amitiés politiques ${ }^{17}$. Ce système donne parfois de bons résultats : même ses adversaires ont rendu hommage à son action. Henri Delaborde, qui jugeait sa nomination injustifiable "du point de vue des droits acquis et des titres", reconnaissait que pendant son passage au Louvre, "il ne laissa pas d'y rendre quelques services" ${ }^{18}$, et Chennevières, après avoir beaucoup médit de son caractère, résumait ainsi le bilan de sa gestion : "II débarrassa le Louvre des expositions annuelles qui pendant quatre mois en cachaient les chefs-d'œuvre; lui étant directeur, furent entrepris et quasi terminés les magnifiques travaux de décoration du grand Salon et de la salle des Sept-Cheminées, et la restauration de la galerie d'Apollon; furent reclassés dans la grande galerie les tableaux de toutes les écoles, furent rédigés et imprimés par Villot, le premier des catalogues modèles de ces tableaux et par Soulié, celui de la chalcographie, tirée la pauvre chalcographie, de sa complète obscurité. Villot commença à surveiller les premiers montages de dessins à la mode de Mariette. On vit peu à peu s'ordonner les antiques et les monuments égyptiens ; et fut vraiment constituée la biliothèque des musées, sous la conduite de Kolloff, le fidèle et savant instrument de Jeanron. Et tout cela en moins de deux ans" ${ }^{19}$.

13 II l'accuse auparavant de s'être attribué la paternité d'un rapport que lui, Chennevières, avait rédigé sur les musées de province, rapport qui provoqua la nomination de quatre inspecteurs, et qui est à l'origine de l'Inspection des Musées classés et contrôlés ${ }^{20}$. Mais le souci qu'avait Jeanron de la bonne conservation des œuvres anciennes se manifeste encore dans un autre rapport, moins connu, du 25 mai 1848, à la suite duquel le ministre de l'Intérieur prit un arrêté interdisant de distraire aucun objet des collections des Musées nationaux, soit pour servir de décoration dans les palais nationaux ou des administrations, soit pour être utilisé comme modèle dans les manufactures nationales ${ }^{21}$.

14 Si l'on en croit Chennevières, Bonaparte aurait, dès le mois de décembre 1848, désigné à la succession de Jeanron le comte de Nieuwerkerke, jeune sculpteur déjà bien en vue ; mais Jeanron aurait su jouer des amitiés et des inimitiés au sein du personnel politique pour se maintenir encore toute une année ${ }^{22}$. La chute de Ledru-Rollin, auquel il devait sa nomination, n'avait par contre été d'aucune conséquence pour sa carrière. Son départ semble donc tenir moins aux liens qu'on aurait pu lui reprocher avec les républicains de la première heure qu'au besoin qu'on avait de son poste pour accorder une faveur.

Son supérieur hiérarchique et rival, le directeur des Beaux-Arts Charles Blanc, connut à peu près le même sort. Révoqué peu après lui, en mars 1850, il dut céder sa place à un ancien journaliste du Globe, de Guizard, qui parvint à la conserver deux ans, jusqu'après le coup d'État, mais qui. "sentant son incompétence, ne fit ni bien ni mal" ${ }^{23}$. Ayant succédé lui-même, le 1er avril 1848, au sculpteur Carraud, qui occupait ces mêmes fonctions depuis le 24 février. Charles Blanc "était amené là, comme on pense bien, par le crédit de son frère" ${ }^{24}$; mais il se maintint, comme Jeanron, bien après que l'évolution politique eut changé de cours. Habileté d'un homme qui savait plaire à ses ministres successifs, ou relative indifférence du pouvoir à l'égard d'une administration chargée, 
certes, d'intérêts prestigieux, mais à peu près dépourvue de toute efficacité sur l'échiquier des luttes politiques? La seconde des deux hypothèses (qui ne s'excluent pas) conduit à s'interroger sur la nature de la considération dont l'art jouissait aux yeux des gouvernants, une question à laquelle il est difficile de répondre, mais sans la réponse à laquelle il est difficile de porter un jugement sur l'action des pouvoirs publics en ce domaine.

La nomination de Charles Blanc et celle de Jeanron eurent pour les artistes une conséquence favorable que souligne Chennevières, qui pourtant ne les aimait pas. L'administration précédente, dit-il, les traitait, sauf les plus grands, avec une insupportable hauteur; "on commença, dès lors, à les respecter et à les tenir pour ce qu'ils valaient" ${ }^{25}$. C'est que Jeanron était peintre lui-même; quant à Charles Blanc, s'il s'était déjà fait à cette époque un nom comme écrivain d'art, il avait d'abord appris la gravure, qu'il pratiqua d'ailleurs longtemps, et appartenait donc au même milieu que ses administres ${ }^{26}$.

En prenant ses fonctions, Charles Blanc n'était pas dépourvu d'idées, loin de là. Il les exposa dans un rapport au ministre de l'Intérieur publié par le Moniteur universel du 10 octobre 1848, dans lequel il réclamait un crédit de 500000 francs pour les arts, égal à celui dont disposait l'ancienne Liste civile. Mais sa demande ne s'appuyait sur aucun projet précis ; c'était, pour reprendre le commentaire qu'en donna L'Artiste du 15 octobre, "un large programme des rêves que fait la République", "une solennelle déclaration de principes" analogue au manifeste adressé par Lamartine à l'Europe après la révolution ${ }^{27}$. En gros, il s'agissait d'un hymne à l'art public, destiné aux masses, sous toutes ses formes, depuis l'architecture des gares jusqu'à la gravure, qui permet une vaste diffusion des images, en passant par la peinture murale, qu'il opposait aux tableaux de chevalet, conçus pour une clientèle particulière, fruits de l'individualisme, production sans grandeur qu'avaient favorisée les régimes précédents. Charles Blanc voyait grand, trop grand sans doute pour les difficultés de l'heure, trop grand surtout pour lui, qui n'était pas un homme d'action, et n'entreprit à peu près rien pour réaliser ses vues généreuses.

Ce n'est pas que de grands travaux n'aient pas été commandés sous son directorat, mais sans programme d'ensemble. Il faut mettre à part la décoration des églises parisiennes, qui se poursuit pendant la Seconde République : l'entreprise dépendait de la Ville, même si l'État y participait financièrement; elle avait commencé sous la Restauration, et se poursuivit jusqu'à la République des ducs. Certes, plusieurs commandes passées dans ce cadre en 1849-1850 ont une importance particulière. Elles sont loin, toutefois, d'égaler en ampleur et en signification celle que reçut Chenavard, en avril 1848, de décorer le Panthéon. C'est à Charles Blanc que revient le mérite, dès son entrée en fonction, d'avoir introduit auprès de Ledru-Rollin le peintre, qu'il connaissait bien, mais l'idée venait --de celui-ci, qui tenait ses esquisses toutes prêtes ${ }^{28}$. La décoration de la galerie d'Apollon, avec le plafond de Delacroix et les tapisseries des Gobelins, entre dans les travaux de restauration du Louvre, entrepris par l'architecte Duban ${ }^{29}$. En matière de sculpture publique, le monument à la mémoire de Mgr Affre, à Notre-Dame, est un ouvrage de circonstance dont l'érection fut décidée par l'Assemblée nationale ; quant aux groupes du pont d'Iéna, les cavaliers grec, romain, arabe et gaulois, c'est l'exécution en pierre de groupes en plâtre commandés pour la Fête de la Concorde ${ }^{30}$. Paradoxalement, Charles Blanc fit commander de nombreux tableaux sans destination précise, ou destinés au musée du Luxembourg, une pratique contraire à ses convictions en matière d'action artistique de l'État; il fit, par ailleurs, exécuter un certain nombre de copies, première 
tentative pour réaliser ce musée des copies qui fut sa principale préoccupation pendant son second directorat, de 1870 à $1873^{31}$.

Un temps, il put craindre que son pouvoir, qu'il exerçait modérément, ne fut menacé. Le 29 octobre 1848, le Ministère instituait auprès de lui une commission permanente des Beaux-Arts, chargée de donner son avis sur l'emploi des crédits et leur répartition, sur les commandes et acquisitions d'ouvrages d'art et leur affectation, sur les secours, les subventions, les souscriptions, sur les écoles d'art et sur les expositions. Elle tint sa première séance le 24 novembre suivant, sa dernière le 12 décembre 1849 mais ne fut officiellement supprimée que par un décret du 7 décembre 1851. En l'absence de procèsverbaux, introuvables aujourd'hui, son activité nous est connue par une courte étude publiée en 1908, et consacrée à la réforme des Salons ${ }^{32}$. Le règlement de celui de 1849 reposa en effet sur le résultat de ses délibérations. Elle ne fut, par contre, jamais consultée sur les commandes et les acquisitions, le directeur des Beaux-Arts (qui n'en devint membre qu'en mai 1849) n'entendant pas se départir de ses pouvoirs en la matière. Autant que nous puissions en juger, son importance tient moins à l'influence, assez restreinte, qu'elle a pu exercer qu'à son existence même. Pour les manufactures nationales, le Ministère de l'Agriculture et du Commerce avait institué le 30 mars 1848 une commission semblable, dite commission supérieure de perfectionnement, qui se réunit plus ou moins régulièrement jusqu'au 18 novembre 1851 et qui joua, elle, le rôle d'un véritable directoire ${ }^{33}$. Malgré la différence profonde entre la situation politique en mars 1848 et au mois d'octobre suivant, les deux créations relèvent du même esprit et préfigurent celles du Conseil supérieur des Beaux-Arts et des commissions de perfectionnement des différentes manufactures sous la Troisième République: elles illustrent la volonté du pouvoir de fonder ses décisions sur les avis de commissions techniques.

20 On aurait pu considérer que telles commissions existaient déjà, sous la forme des différentes classes de l'Institut. C'était, en théorie du moins, l'une de leurs fonctions, fonction dont l'Académie des Beaux-Arts revendiqua l'exercice tout au long du siècle. Le Ministère de l'Instruction publique s'en souvint dans les premiers jours qui suivirent la révolution de février. Il voulut alors remettre en vigueur une disposition prescrivant a l'Institut de nommer tous les ans six de ses membres pour entreprendre des voyages de recherche sur les différentes branches des connaissances humaines autres que l'agriculture. L'Académie des Beaux-Arts envisagea dans le cadre de ces missions l'étude des collections d'art et des monuments historiques, de leur conservation et de leur usage, et celle des écoles d'art et des améliorations qui pourraient leur être apportées ${ }^{34}$. Mais l'affaire ne semble pas avoir connu de suite.

21 L'Académie des Beaux-Arts régnait de droit sur la villa Médicis, et de fait (en particulier par le jugement du concours pour le prix de Rome) sur l'École des Beaux-Arts. Dans les derniers jours de son directorat, Carraud fit créer une commission chargée d'étudier une réforme de ces deux institutions. Elle se choisit pour président le peintre Horace Vernet, qui assumait aussi à l'époque la présidence de l'Académie. Très vite, à propos du jury des concours, un désaccord apparut entre ses membres, provoquant le départ des minoritaires et la dissolution d"'une commission, qui n'avait été instituée que pour examiner quelles étaient les réformes à faire et qui de prime abord se refusait à toute réforme", comme l'écrivait Charles Blanc dans un rapport au ministre, vers la fin avril ${ }^{35}$. La tournure prise par les choses était prévisible : l'échec reproduisait très exactement celui de la commission chargée en 1831 par Montalivet de la même tâche ${ }^{36}$. 

s'effacer devant celle du vaste mouvement suscité par la révolution de février en faveur de la création d'une assemblée générale des artistes, qui aurait assumé démocratiquement la conduite de leurs affaires. L'histoire de ce mouvement est loin d'être éclaircie dans tous ses épisodes, et peut-être ne le sera-t-elle jamais. Du moins peut-on essayer d'en résumer les grandes lignes. Dès la proclamation de la République, Barye, Couture et Diaz adressèrent au gouvernement provisoire, au nom de leurs confrères, une pétition pour réclamer que "les fonctionnaires qui, par la nature de leur emploi, exercent une action immédiate et directe sur les beaux-arts soient élus par la corporation des artistes en assemblée générale" ${ }^{37}$. Une autre pétition des artistes concernait l'autorisation de se réunir en assemblée générale et d'élire un comité chargé de s'occuper des grandes questions relatives à l'organisation des beaux-arts ${ }^{38}$. Après accord du gouvernement, elle fut suivie d'une nouvelle pétition, en date du 2 mars, pour réclamer une salle de réunion ${ }^{39}$.

En réalité, un malentendu existait déjà entre les artistes et le gouvernement. Par un arrêté du 29 février, celui-ci les avait bien convoqués pour le 5 mars, mais il n'avait fixé d'autre tâche à cette assemblée que d'élire une commission chargée du placement des œuvres au Salon dont l'ouverture était prévue pour le 15. Certains d'entre eux ayant voulu profiter de cette occasion pour élire un comité représentant les intérêts des arts, les "délégués provisoires des artistes du dessin" demandèrent au ministre de rappeler le but précis de la réunion du 5 et d'avertir qu'une autre convocation suivrait en vue de constituer l'assemblée permanente des artistes ${ }^{40}$.

Une assemblée, finalement, se tint le 21 avril au Palais Bourbon, sous la présidence du peintre Decamps ${ }^{41}$. Elle ne réunissait pas tous les artistes, mais seulement les peintres, et rien n'indique que l'initiative en soit venue de l'administration. On sait toutefois que Charles Blanc y parut, et qu'il monta à la tribune "pour annoncer l'intention du ministre de prendre en grande considération les vœux des artistes tout en sauvegardant l'autorité déposée entre ses mains" ${ }^{42}$. Un comité de 72 membres y fut alors élu, qui constitua le 30 avril son bureau ${ }^{43}$. Peu après, six membres du bureau signaient une lettre au ministre pour lui proposer le concours du comité dans toutes les occasions touchant aux intérêts de l'art et des artistes ${ }^{44}$. Une demande plus précise, formulée un peu plus tard, concernait les récompenses à décerner par l'État: les peintres désiraient qu'elles le fussent par un jury nommé par les artistes et suivant des dispositions arrêtées par eux. Elle reçut le 26 mai une réponse négative, signée par le ministre, mais rédigée par Charles Blanc : "Les membres du Comité de peinture ont trop d'expérience et j'ai trop de confiance dans leurs lumières et dans leur haute intelligence pour croire qu'ils voulussent appuyer des prétentions dont l'exigence ne tendrait à rien moins qu'à dicter les résolutions dont le Ministre doit seul rester le maître, parce que seul il connaît les ressources dont il lui est permis de disposer et que seul il est responsable de leur emploi devant l'assemblée souveraine" ${ }^{45}$.

Les journées de juin et l'état de siège mirent en sommeil l'action du comité de peinture, mais il parvint à rédiger un projet de constitution qui fut présenté aux peintres lors d'une assemblée générale organisée a l'Institut le 7 janvier 1849, et approuvé par une seconde assemblée qui se tint le 10 février suivant au Conservatoire des Arts et Métiers ${ }^{46}$. Dans le même temps, les sculpteurs et les graveurs avaient élaboré de leur côté deux projets analogues, sur le contenu desquels nous sommes aussi peu renseignés que sur celui des peintres. Il est à peu près certain que ces textes restèrent lettre morte. 

principe électif, qui en était le critère et le garant. On sait ce que furent les luttes pour la conquête ou l'élargissement du droit de vote dans la première moitié du XIX siècle. La volonté de faire triompher en tout et partout ce principe caractérise l'esprit du temps. C'est en son nom que les délégués de la section de peinture protestèrent en 1848 contre la création d'une commission chargée d'étudier les réformes à apporter à l'École des BeauxArts et à la villa Médicis, parce qu'il s'agissait d'une décision administrative ${ }^{47}$; c'est encore en son nom que, lors de la-première séance de la commission de perfectionnement des manufactures nationales, certains de ses membres, des artistes, s'interrogèrent sur la légitimité de leur présence, du fait qu'ils avaient été nommés par le ministre, et non élus par leurs confrères ${ }^{48}$. inspirer certaines interprétations, comme celle de T. J. Clark, qui dénonce dans les termes du refus opposé par le ministre, le 26 mai 1848, à une demande du bureau du comité des 72, "the language of reaction, polite, vague, completely obstructive" ${ }^{49}$. À cette lettre, il oppose la généreuse réponse qu'en mars, Armand Marrast, au nom du gouvernement provisoire, aurait faite aux artistes: "Organisez-vous vous-mêmes" ${ }^{50}$. Flux de l'esprit démocratique, donc, puis reflux, retour à l'ordre ancien. Mais la déclaration d'Armand Marrast faisait suite à une demande adressée par les artistes au gouvernement, de bien vouloir les réunir en assemblée, Le droit d'association venant d'être proclamé avec la République, il leur rappelait qu'ils étaient libres d'en former une sans avoir à en demandera l'autorisation, ni à solliciter de l'État qu'il en prenne l'initiative : “Nous n'avons ni à vous réunir, ni à vous constituer, organisez-vous vous-mêmes". Cela ne préjugeait en rien du départ des compétences respectives entre cette association et le gouvernement, comme Charles Blanc le laissa entendre à l'assemblée du 21 avril, et là réside le vrai problème.

La position des artistes aurait été plus solide si, voulant assumer eux-mêmes le pouvoir de décision dans toutes les affaires concernant l'art, ils avaient fait valoir qu'elles n'intéressaient qu'eux et leurs rapports avec le public, que l'État n'avait pas à s'en mêler, bref, s'ils s'étaient prononcés pour cette "séparation de l'Art et de l'État" que certains réclamèrent un demi-siècle plus tard, à une époque où le désengagement constituait le fondement de la doctrine officielle en la matière ${ }^{51}$. Bien au contraire, formés à la longue tradition du mécénat royal, puis impérial et même républicain, et y trouvant peut-être aussi leur avantage, ils ne contestaient pas l'intervention de l'État, que ce soit dans l'organisation des expositions annuelles que par les achats et la distribution de récompenses. Ce faisant, ils pouvaient demander au gouvernement d'écouter leur avis dans les questions techniques, mais non, comme représentants d'une profession, disposer à sa place de l'utilisation des fonds publics, dont il est, selon le principe même du régime parlementaire, responsable devant la représentation nationale: tel était le sens de la lettre adressée par le ministre au comité des 72 le 26 mai. Il est probable que, confronté à une demande similaire, Armand Marrast leur eût opposé la même fin de non recevoir.

En réalité, même si l'on n'attache pas de signification particulière à l'emploi, courant à l'époque, du terme de corporation, hérité de l'Ancien Régime, et que remplace parfois l'expression de République des Arts, il semble que ce soit, très confusément, un idéal de société corporatiste qui ait inspiré les revendications des artistes. Or, moins encore que l'évolution générale de la société française, celle de leur propre métier ne favorisait Pas la réalisation d'un tel idéal. Une association corporative ne se maintient que si le métier possède une définition tant soit peu précise, et ses membres suffisamment d'intérêts 
communs à défendre, ce qui n'était plus le cass au milieu du XIX siècle. Les critères retenus en 1848 pour établir la qualité d'artiste en vue d'une assemblée générale (qui restait, au demeurant, exclusivement parisienne) étaient trop larges et trop formels Pour que pût en résulter une solidarité véritable ${ }^{52}$. Sauf à ce que l'État lui confère ce monopole dont jouissait l'ancienne Académie royale de peinture et de sculpture, ce qui était impensable à l'époque, une association des Listes autre qu'une simple société de secours mutuels comme celle que le baron Taylor avait fondée en 1844 (et qui se maintint parce qu'elle ne se proposa jamais d'autres buts) était vouée à plus ou moins brève échéance à l'éclatement et à la concurrence d'associations rivales, comme cela se produisit après 1880 , lorsque les artistes furent contraints de s'organiser pour assurer la tenue des Salons ${ }^{53}$.

La volonté de démocratie qu'ils affichèrent au début de la Seconde République est évidemment liée aux événements, plus précisément à la conquête du suffrage universel. Il s'agit là d'un droit purement politique dont la revendication ne permet pas de distinguer les républicains sociaux de février des républicains modérés de mai. La liste des peintres qui formaierrt le bureau du comité des 72 montre bien que les limites de leur engagement politique : si tous ne partageaient pas les mêmes sentiments, ni Ingres, ni Delacroix, ni Fiandrin, ni Lehmann ne peuvent passer pour de farouches démocrates, en dépit d'un ralliement sincère au régime ${ }^{54}$. Plus révélateur encore est l'exemple de Nieuwerkerke, qui, devenu directeur des Beaux-Arts, le resta pendant tout l'Empire (avec même, à partir de 1863, le titre de surintendant): sa signature se rencontre, avec celle des autres délégués des sculpteurs, au bas de la protestation du 6 avril 1848 contre la nomination par voie administrative d'une commission chargée d'étudier la réforme de l'École des BeauxArts, et de quelques autres textes de tendance analogue rédigés par les mêmes délégués ${ }^{55}$.

De toutes les affaires dont s'occupait l'administration des Beaux-Arts, il en est une qui retenait particulièrement l'attention des artistes et de l'opinion publique, à laquelle les principes de la vie politique semblaient s'appliquer directement et sur laquelle la révolution de février eut des conséquences immédiates et considérables en apparence : l'organisation des Salons annuels. Par le même arrêté du 24 février qui réorganisait la direction des Beaux-Arts, Ledru-Rollin décidait que celui de 1848 serait ouvert le 15 mars, et que le jury chargé de recevoir les œuvres (le texte, par un lapsus significatif de l'importance particulière qu'avait la peinture, dit "les tableaux") serait élu par les artistes. Cinq jours plus tard, un nouvel arrêté rendait libre l'admission pour tous les ouvrages envoyés; il convoquait par ailleurs les artistes pour le 5 mars afin d'élire une commission de placement --mesure qui, nous l'avons vu, suscita de la part de certains d'entre eux une interprétation erronée.

Cette liberté totale d'admission n'était pas nouvelle ; elle avait déjà régné à partir de 1791, jusqu'à ce que les protestations des artistes eux-mêmes entrainent en 1798 la création d'un jury. L'aspect du Salon de 1848, où le nombre des ouvrages exposés atteignait 5 180, conduisit le gouvernement à rétablir l'année suivante le jury, mais un jury élu par tous les artistes, en apparente conformité avec le principe de la démocratie.

L'importance de ces mesures ne se comprend que si l'on se rappelle les scandales provoqués tous les ans, sous la monarchie de Juillet, par les proscriptions dues à un jury composé des membres de l'Académie des Beaux-Arts ${ }^{56}$. On trouvera dans le livre de Rosenthal déjà cité des exemples des protestations annuelles, et de remèdes proposés ${ }^{57}$. Un petit livre anonyme, intitulé De l'Exposition et du jury, mérite une attention particulière, car, publié tout au début de l'année 1848, il passe pour avoir inspiré le système adopté 
l'année suivante. Dû à la collaboration de Boissard, Villot et Clément de Ris, il reprenait dans sa première partie une plaquette rédigée par ce dernier en vue du Salon de 1847, et présentée sous un titre on ne peut plus significatif : De l'oppression dans les Arts. L'auteur y prônait un jury de vingt membres élus, dont dix choisis dans la quatrième classe de l'Institut. Cette clause restrictive disparut de l'ouvrage suivant, dont les auteurs, tout en insistant sur la nécessité d'un jury d'admission, le voulaient librement élu par tous les artistes ayant exposé depuis 1830 .

L'essentiel, cependant, ne résidait pas dans les détails du règlement proposé, mais dans le ton, dans la dénonciation de "l'oppression qui pèse depuis tant de siècles sur les artistes" (p. III), du "pouvoir tyrannique" qui "impose sans appel des lois émanées de son bon plaisir à une corporation tout entière" (p. 1). Le rapprochement s'imposait avec la lutte menée contre les abus de l'Ancien Régime. Aussi les mesures prises en 1848 et 1849 semblent-elles s'opposer à l'arbitraire précédent comme la république s'oppose à la monarchie. Parallèle évident, mais en grande partie fallacieux.

En confiant l'admission des œuvres à l'Académie des Beaux-Arts, qui pouvait passer, a priori, pour la plus haute autorité en la matière et qui jouissait d'une indépendance certaine à l'égard du pouvoir, le gouvernement de la monarchie de Juillet avait sans doute voulu dégager sa responsabilité, ou la responsabilité de l'administration. D'ailleurs, les proscriptions du jury frappèrent souvent, et comme à dessein, des peintres qui jouissaient de la faveur des bureaux. Son arbitraire, qu'aggravait cette circonstance particulière, souvent dénoncée, que les décisions étaient imposées par quelques architectes aussi ignorants de peinture qu'assidus aux séances, en l'absence chronique d'une majorité des peintres, ne saurait donc s'assimiler directement à l'autoritarisme de la monarchie. Bien plutôt résulterait-il de la conjonction du principe (électif) de cooptation qui est encore celui des Académies comme de la quasi totalité des sociétés savantes, et d'une doctrine artistique (le néo-classicisme) très exclusive --mais dont le caractère exclusif tenait moins à son contenu proprement esthétique (on peut sans intolérance aucune juger le dessin supérieur à la couleur) qu'au contexte moral et politique dans lequel elle était apparue et s'était affirmée, c'est-à-dire, paradoxalement, l'esprit des Lumières et les luttes de la Révolution.

Reposant sur une analyse superficielle, sur l'illusion des mots, l'assimilation entre le mode de recrutement du jury d'admission et le principe du régime politique devait aussi conduire à une solution fausse, qui a pu passer pour idéale, mais qui n'a fait que perpétuer les contradictions dont souffrait le Salon ${ }^{58}$. Pour les uns, celui-ci devait être une exposition choisie, de prestige, destinée à montrer par la réunion de ce que les artistes avaient produit de meilleur, les progrès accomplis par l'école française. D'où la nécessité d'un jury sévère, ce dont la majorité des exposants ne voulaient pas, d'un jury dont aucun mode de recrutement ne pouvait assurer parfaitement la compétence, mais dont la nomination semblait devoir revenir à l'État, dans la mesure où il assumait la charge de son organisation. Pour d'autres, au contraire, le Salon n'était plus qu'un marché d'œuvres d'art, un bazar, pour reprendre un mot dont l'usage se perpétua tard dans le siècle ${ }^{59}$. C'est l'opinion qu'Ingres, qui n'exposait plus depuis longtemps, défendit devant la Commission permanente des Beaux-Arts ${ }^{60}$. Mais rien, dans ce cas, comme il le fit valoir, ne justifiait l'existence d'un jury, n'autorisait à empêcher quiconque d'offrir sa production à la vue d'éventuels acheteurs. Il fallait par contre en tirer cette conséquence que son organisation ne relevait pas de la compétence des pouvoirs publics et que c'était aux artistes de s'entendre pour s'occuper de leurs propres affaires. Cette idée, qui 
commence à se manifester à l'époque, se confond souvent avec la notion d'exposition permanente, que l'on retrouve sous de nombreuses plumes, avec des acceptions diverses, jusqu'au début de la Troisième République. Elle se rencontre en particulier dans le rapport adressé par Charles Blanc au ministre de l'Intérieur et publié le 10 octobre $1848{ }^{61}$. En regard de ces conceptions divergentes, mais cohérentes, la solution adoptée en 1849 était une solution bâtarde logique dans le cadre d'une société d'artistes organisant sa propre exposition, mais qui, appliquée au Salon, se révéla par la suite incapable d'en résoudre les problèmes.

Une autre réforme eut plus de poids que celle du jury. Depuis son origine, le Salon se tenait au Louvre. Il en résultait une gêne considérable, et des risques renouvelés pour les tableaux anciens, visibles six mois par an, compte tenu de la longueur des travaux d'installation et de démontage. En 1849, l'administration, sur les instances de Jeanron, décida de le déplacer. Il eut lieu cette année-là aux Tuileries, puis au Palais national (le Palais royal) l'année suivante. La portée de ce changement n'était pas seulement d'ordre matériel. Certains contemporains tenaient la présentation d'œuvres modernes dans le musée du Louvre pour un symbole du maintien de la tradition. Cette manière de voir impliquait un choix sévère, qui ne laissât pénétrer dans le temple de l'Art que les œuvres susceptibles de supporter dignement la comparaison avec celles qui s'y trouvaient à demeure. Dans cette optique, l'abandon de ce lieu confirmait la transformation du Salon en bazar, c'est-à-dire en foire et en divertissement mondain, une évolution que déplorait déjà Balzac dans Pierre Crassou dix ans plus tôt, et qui, à terme, devait provoquer son déclin.

La révolution, l'établissement de la République suscitèrent de grandes espérances dans le domaine des arts. La revue L'Artiste s'en est fait l'écho à plusieurs reprises, en particulier avec un article de Théophile Gautier sur "L'Art en 1848", paru dans le numéro du 15 mai, et un autre, de Charles Isnard, publié le $1^{\mathrm{er}}$ octobre sous le titre "De la peinture la veille et le lendemain de la République". Les deux auteurs prônaient, comme Charles Blanc dans son rapport au ministre de l'Intérieur, la décoration peinte des édifices publics, parce qu'il s'agit d'un art pour le peuple, destiné a l'instruire, à le fortifier dans les vertus civiques, à cimenter son unité.

Mais cette mission attribuée à la peinture monumentale, comme à la sculpture du même ordre, le fut sous tous les régimes du XIX ${ }^{e}$ siècle, et encore par certains régimes du XXe, avec une simple différence dans la coloration politique. Pour le reste, les intérêts, les opinions diffèrent, comme si, par delà l'enthousiasme commun provoqué par l'avènement de la république, chacun avait attendu d'elle la réalisation de ce qui lui tenait particulièrement à cœur, et qui ressortissait souvent à une conception très traditionnelle de l'art. Lacune symptomatique : ces auteurs qui parlent de grande peinture et de paysage ne semblent s'intéresser ni à l'artisanat, ni à l'art industriel. N'était-ce pas de là, pourtant, qu'allaient venir les changements les plus profonds? 


\section{NOTES}

1. Ces pages reprennent, en la précisant, la première partie d'une communication sur la politique artistique des pouvoirs publics sous la Seconde République présentée en 1981 devant la Société d'Histoire de la Révolution de 1848.

2. Arch. nat., F21 23. Voir aussi Pierre Angrand. "L'Administration des Beaux-Arts et l'œuvre de Daumier", La Pensée, n 177, octobre 1974, ainsi que le livre de T. J. Clark cité note 10 (p. 108-111).

3. Arch. nat. F21 496. Près de la moitié de ce crédit devait être dépensé en secours, encouragements, subventions. Les achats et commandes de peinture s'élevaient à 68600 francs. Aucun tableau ne dépassait le prix de 1500 francs ; la commande à Daumier s'élevait à 1000 francs. La minute d'une réponse du ministre de l'Intérieur au Président du Comité de l'Intérieur concernant un programme de travaux artistiques que celui-ci lui avait soumis apporte quelques précisions sur l'emploi de ce crédit : “A peine suffisant pour apporter quelques soulagements aux misères les plus pressantes, il ne peut être dépensé, ainsi que le chef du Pouvoir exécutif et le Conseil des Ministres l'ont décidé, que par petites sommes. Ce fonds, d'une nature toute spéciale, doit être d'ailleurs dépensé dans l'année, ne peut être reporté d'un exercice sur l'autre et ne doit, par conséquent, être appliqué qu'à des encouragements et à des commandes d'un ordre secondaire ; les règles de la comptabilité s'opposant à ce que l'on impute une même dépense sur différents crédits, il ne pourrait être employé aux paiements d'acomptes, comme l'indique la note du comité". C'est pourtant ce qui se produisit avec la commande à Daumier!

4. Tout l'œuvre peint de Daumier, coll. Les Classiques de l'Art, Paris, 1972, cat. $\mathrm{n}^{\circ} 38$ (esquisse pour le tableau).

5. Michael Marrinan, "Resistance, Revolution and the July Monarchy : Images to Inspire the Chamber of Deputies", Oxford Art Journal, III/2, octobre 1980, p. 26-37. Sur les versions qui en furent données entre 1939 et 1981, voir la "Note bibliographique" p. 36.

6. Léon Rosenthal, Du Romantisme au Réalisme. Essai sur l'évolution de la peinture en France de 1830 à 1848, Paris, 1914, p. 7.

7. François Benoît, L'Art français sous la Révolution et l'Empire, Paris, 1897 (réédition anastatique, Genève, 1975) ; René Schneider, Quatremère de Quincy et son intervention dans les arts, Paris, 1910.

8. Les résultats des différents concours sont présentés par Maurice Agulhon dans son livre Marianne au combat. L'imagerie et la symbolique républicaine de 1789 à 1880, Paris, 1979, p. 97 et suivantes ; mais il s'agit d'un ouvrage consacré, comme son sous-titre l'indique, à l'imagerie politique. La principale étude sur le concours pour la figure peinte est celle d'Albert Boime, The Second Republic's Contest for the Figure of the Republic, The Art Bulletin, March 1971, p. 68-83. Il est symptomatique que T. J. Clark (ouv. cité infra, note 10) signale les trois concours (p.63) mais ne commente que celui pour la figure peinte, et cela pour souligner (p. 64) l'originalité de l'esquisse de Daumier face à la monotonie de celles des autres concurrents, qui se limitaient pour la plupart à une seule figure allégorique -- ce qui était... une condition spécifiée dans le programme du concours ! Sur le concours pour 
la figure sculptée, voir Jacques Lethève, "Une statue malchanceuse, "La République"” de Jean-François Soitoux, Gazette des Beaux-Arts, octobre 1963. p. 229-240.

9. Il faut rendre ici hommage à Pierre Angrand pour son rôle de pionnier, ainsi que pour ses nombreuses publications fondées sur le dépouillement des archives de l'ancienne Direction des Beaux-Arts.

10. T. J. Clark, The Absolute Bourgeois. Artists and Politics in France 1848-1851, Londres. 1973. chap. 2. "The Art of the Republic", pp. 31-72.

11. En dehors de cette phrase, l'arrêté du 24 février concernait l'organisation du Salon de 1848. Voici le texte de l'arrêté du 18 mars 1848 : “1) Les musées du Louvre, du Luxembourg, de Versailles, les galeries des anciennes résidences royales et palais du gouvernement, sont distraits de l'administration de la liste civile pour rentrer dans les attributions du ministère de l'Intérieur. $2^{\circ}$ ) Le ministre de l'Intérieur fera rechercher les objets d'art appartenant à l'État qui auraient été déplacés et qui se trouveraient compris indûment dans le domaine privé, ainsi que ceux qui se trouveraient dans les établissements publics quels qu'ils soient. $3^{\circ}$ ) Les trois manufactures de Sèvres, des Gobelins et de Beauvais rentrent dans le département de l'agriculture et du commerce". 12. François Benoît, ouv. cité (note 7), p. 146-147.

13. Le texte en est reproduit par Philippe de Chennevières, Souvenirs d'un Directeur des Beaux-Arts, $2^{e}$ partie, Paris, 1886, p. 63 (réédition anastatique, Paris, Arthéna, 1979). Un peu plus tard, la Société libre des Beaux-Arts adressait à l'Assemblée nationale une pétition dans laquelle ce rattachement était aussi demandé, ainsi que celui des manufactures nationales au ministère des Travaux publics [L'Artiste, $\mathrm{V}^{\mathrm{e}}$ série, tome $\mathrm{I}$, 15 juillet 1848, p. 208).

14. L'Artiste du $1^{\mathrm{e}}$ janvier 1849, "Mouvement des arts", par Lord Pilgrim : “Avant d'être nommé président, M. Louis-Napoléon Bonaparte avait eu l'idée d'un ministère des Arts et des Lettres. On aurait détaché à l'Intérieur la division des Beaux-Arts, à l'Instruction publique la division des Lettres : rien de plus intelligent. Déjà on avait, il y a deux ans, pensé à ce ministère pour $\mathrm{M}$. Vitet. Mais, en France, on a peur du nouveau comme de l'inconnu" (p. 146). Le ministère des Beaux-Arts fut créé par décret du 2 janvier 1870 ; il prit le titre de ministère des Lettres, Sciences et Beaux-Arts par décret du 15 mai 1870. 15. Pierre Vaisse, Le Conseil Supérieur de Perfectionnement des manufactures nationales sous la Deuxième République, Bulletin de la Société de l'Histoire de l'Art Français, Année 1974, Paris, 1975, p. 153-171.

16. Si l'on en croit Philippe de Chennevières, Charles Blanc était "mal disposé pour Jeanron par les perpétuels tiraillements de leurs deux directions" (ouv. cité (note 13), $2^{\mathrm{e}}$ Partie, 1885, p. 85) ; il dit ailleurs que "ce qui agaçait le plus Jeanron dans son départ, c'était que Charles Blanc lui survivrait" (Ibidem. $3^{\text {e }}$ Partie, p. 79).

17. Philippe de Chennevières, Ibidem., $3^{\mathrm{e}}$ Partie, p. 60.

18. Henri Delaborde, L'Académie des Beaux-Arts depuis la fondation de l'Institut de France, Paris, 1891, p. 281.

19. Ouv. cité (note 13), p. 83.

20. Ibidem., p. 68-70. Le rapport de Jeanron date du 3 avril 1848. Voir Harsène Houssaye, “Les Musées de Province”, L'Artiste du 16 avril 1848, p. 89-90.

21. L'arrêté du ministre de l'Intérieur date du 4 juin 1848. Il dispose qu"'aucun objet ne pourra être distrait des Musées nationaux, soit pour servir de décoration, soit pour être reproduit dans les Établissements spéciaux. Pour ce dernier objet, les manufactures publiques recevront, dans les Musées mêmes, toutes les facilités nécessaires pour établir 
les copies ou cartons qui pourront leur être utiles, le tout sous la surveillance des conservateurs compétents".

22. Ouv. cité (note 13), p. 78.

23. Ibidem, $2^{\mathrm{e}}$ Partie, p. 55.

24. Ibidem,. p. 54.

25. Ibidem.

26. Sur Charles Blanc, voir e. a., Philippe de Chennevières, Souvenirs d'un Directeur des Beaux-Arts, $1^{\mathrm{e}}$ Partie, 1883, p. 86-98; Tullo Massarani, Charles Blanc et son œuvre, Paris, 1885 ; A. Soubies, Les membres de l'Académie des Beaux-Arts depuis la fondation de l'Institut, $3^{\mathrm{e}}$ série, 1852-1876, Paris, 1911, tome 3, p. 228-233; et le catalogue de l'exposition Hommage à Charles Blanc, Castres, musée Goya, 1948.

27. L'Artiste du 15 octobre 1848, p. 65.

28. Pour cette commande, il faut se reporter au récit qu'en a donné Philippe de Chennevières. Souvenirs d'un Directeur des Beaux-Arts. Les décorations du Panthéon, Paris, 1885, p. 62 et suivantes. L'ambitieux programme de Chenavard a été décrit ou étudié par Théophile Gautier, L'Art moderne, Paris, 1856, p. 59-94 (réédition d'un article de septembre 1848, paru dans La Presse) ; Gustave Planche, "Les cartons de M. P. Chenavard", Revue des Deux Mondes, XIII (1852/1), p. 362-377 ; Joseph C. Sloane, Paul Chenavard, Art Bulletin, décembre 1951, p. 240-258; idem, Paul Marc Joseph Chenavard, Artist of 1848, Chapel Hill, The University of North Carolina Press, 1962 ; M. A. Grunewald, Paul Chenavard et la décoration du Panthéon de Paris en 1848, Lyon, Musée des Beaux-Arts, 1977 ; idem, "Paul Chenavard (1807-1895), La Palingénésie sociale ou la Philosophie de l'histoire (1830 ? -- 1852 ?)”, Bulletin des Musées lyonnais, 1980/1, p. 1-27.

29. Philippe de Chennevières, Notice historique et descriptive sur la galerie d'Apollon au Louvre, Paris, 1851, rééd. 1855, p. 65 sqq.

30. Pour le monument à la mémoire de Mgr Affre, le vote de l'Assemblée nationale eut lieu le 17 juillet 1848. A la suite d'un concours, la commande fut passée au sculpteur Auguste Debay, pour la somme de 49000 francs (Arch. nat., F21 42). Les groupes du pont d'Iéna furent commandés à Daumas (F21 23), Devaulx (F21 25), Feuchère (F21 29 B), Préault (F21 51). La commande des modèles en plâtre date du 12 mai 1848 ; elle s'élevait à 7000 francs par artiste, pris sur le crédit de 950000 francs ouvert pour la Fête de la Concorde par un décret du gouvernement provisoire en date du 27 avril. Celle des groupes en pierre fut signée le 21 septembre 1849, à raison de 22000 francs l'un ; leur mise en place était achevée le 15 juin 1853.

31. Pierre Vaisse, "Charles Blanc und das "Musée des copies"“", Zeitschrift fur Kunstgeschichte, 1976/1, p. 54-66.

32. Jean-Louis Fouché, "L'Opinion d'Ingres sur les Salons. Procès-verbaux de la Commission permanente des Beaux-Arts (1848-1849)", Chronique des Arts et de la Curiosité, 14 mars 1908, p. 98-99 et 4 avril 1908, p. 129-130. La liste des membres donnée p. 98 diffère un peu de celle que l'on trouve dans l'Almanach national 1848-1849-1850, p. 136 : Ingres avait donné sa démission, et d'Albert de Luynes, Charles Blanc et le député Frémy avaient été nommés. Le décret du 7 décembre 1851 supprimait aussi la Commission permanente des Théâtres, instituée par le même arrêté du 29 octobre 1848.

33. Pierre Vaisse, ouv. cité, supra (note 15).

34. P.-v. de l'Académie des Beaux-Arts, séances des 4 et 18 mars 1848 (Arch. de l'Institut, $2 \mathrm{E}$ 10).

35. Ibidem, séance du $1^{\mathrm{er}}$ avril 1848, et Henri Lapauze, L'Académie de France à Rome, Paris, 1924, II, p. 292 et suivantes. 
36. Léon Rosenthal, ouv. cité (note 6), p. 5.

37. Arsène Houssaye, “République des Arts et des Lettres”, L'Artiste des 25 février et 5 mars, p. 258. Les trois artistes envoyèrent (ou projetèrent d'envoyer) aux journaux qui avaient fait état de leur pétition une lettre pour préciser qu'ils n'avaient été que "l'écho de la pensée générale" (Bibl. Doucet, carton 36, sculpteurs -- Barye). Voir aussi T. J. Clark, ouv. cité (note 10), p. 50.

38. Arch. nat., F21 527, "Communication officieuse des délégués des artistes" : "La majorité des artistes peintres, sculpteurs, etc., parmi laquelle on remarque les plus grands noms, ont adressé une pétition au gouvernement provisoire à l'effet d'avoir l'autorisation de se réunir en assemblée générale. Dans cette assemblée générale, un comité serait nommé et s'occuperait des grandes questions relatives à une organisation large des beaux-arts. Leur travail serait communiqué au gouvernement. Le gouvernement provisoires a accueilli cette pétition avec sympathie et a invité les artistes à se réunir le plus tôt possible. Les mêmes artistes demandent donc au ministère de l'Intérieur qu'un local soit mis à leur disposition. Ils ont hâte de se constituer en société permanente et de nommer un comité afin qu'il soit procédé sans retard aux mesures que réclame l'état actuel des beaux-arts".

39. Ibidem., Cette pétition était signée, entre autres, par Rousseau, Delacroix, Picot, Muller, Ingres, Delaroche, Ary Scheffer, Jeanron, Barye, Diaz, Couture.

40. Ibidem, Le texte de l'arrêté du 29 février figure dans le livret du Salon de 1848. Voir aussi Chennevières, ouv. cité (note 13), p. 62.

41. La convocation parut dans L'Artiste du 16 avril 1848. p. 91. Voir aussi Paul Mantz, Decamps, Gazette des Beaux-Arts, février 1862, p. 122.

42. L'Artiste du 30 avril 1848, p. 110.

43. Le bureau était ainsi constitué : Présidents : Delaroche (Paul), Ingres, Delacroix (Eugène), Decamps ; Vice-Présidents : Cogniet (Léon), Nanteuil (Célestin), Drolling, Flandrin (Hippolyte), Leleux (Armand), Corot ; Secrétaires : Pérignon, Dauzats, Lehmann (Henri), Lefevre (Charles), Boissard, Lazerges, Billotte, Cérôme, Aligny, Jollivet, Ouvrié (Justin), Timbal, Riesener (Arch. nat., F21 566).

44. Ibidem., Fac-similé de la lettre dans T. J. Clark, ouv. cité (note 10), p. 53.

45. Ibidem.

46. L'Artiste du 15 janvier 1849, p. 163-164, et du 15 février, p. 188-191.

47. Arch. nat., F21 566, lettre du 6 avril 1848. Premier signataire, en tant que président. Rude avait été nommé par le pouvoir pour faire partie de la commission. Selon T. J. Clark, ouv. cité (note 10), p. 50, la lettre aurait été rédigée "in protest at the new regulations for the Arts, which put an end to improvisation and the power of Jeanron, and put Museums and Fine Art under separate management" : elle ne contient, en réalité, aucune allusion à ces questions et ne concerne que "l'arrêté du 30 mars qui nomme une commission chargée des réformes à faire à l'organisation de l'école française de Rome et de l'école des Beaux-Arts".

48. Pierre Vaisse. ouv. cité (note 15), p. 155-156. C'est Delaroche qui ouvrit le débat sur cette question.

49. T. J. Clark, ouv. cité (note 10), p. 51.

50. Réponse rappelée par les délégués des sculpteurs dans leur lettre du 6 avril [supra, note 47). Ils l'interprétaient (abusivement) comme une reconnaissance de la compétence des commissions formées par les artistes au sein des différentes sections pour étudier les réformes à faire, donc comme un argument contre la nomination d'une commission par le gouvernement. 
51. Voir l'Enquête sur la Séparation des Beaux-Arts et de l'État, publiée par Les Arts de la Vie, octobre 1904.

52. L'Artiste du 16 avril 1848, p. 91 : “Les peintres qui désireraient se faire inscrire comme électeurs devront justifier de leur qualité d'artistes par une preuve d'admission aux expositions du Louvre antérieures et non compris celle de 1848, ou par une médaille ou un prix obtenu à l'École des Beaux-Arts, ou enfin ceux qui n'auraient aucun de ces moyens d'admission seront tenus de présenter la signature de quatre peintres exposants, au moins, répondant de leur capacité comme électeurs".

53. Pierre Vaisse, Salons, expositions et sociétés d'artistes en France 1871-1914, Saloni, Callerie, Musei e loro influenza sullo sviluppo dell'arte dei secoli XIX e XX, a cura di Fracis Haskell, Atti del XXIV Congresso Internazionale di Storia dell'Arte, volume 7, Bologna, s. d., p. 141-155. 54. Les sentiments d'Ingres et de Delacroix sont bien connus. Sur Hippolyte Flandrin, voir Louis Flandrin, Hippolyte Flandrin. Sa vie, son œuvre, Paris, 1902, p. 190 sqq., et Michael Paul Driskel, Painting, Piety, and Politics in 1848 : Hippolyte Flandrin's Emblem of Equality at Nîmes, Art Bulletin, June 1984, p. 270-285.

55. Arch. nat., F21 566 : lettres des délégués de la section de sculpture des 23 mars (et non février, comme il est écrit par erreur) et 11 avril 1848 relatives à l'organisation du concours pour la figure sculptée de la République et au mode de désignation du jury (les délégués réclament qu'il soit élu).

56. Les musiciens en furent toutefois exclus à partir d'octobre 1833.

57. Ouv. cité (note 6), p. 38 sqq.

58. Cette illusion s'est perpétuée longtemps. Voir, par ex. Germain Bazin, Le Salon de 1830 à 1900, Scritti di storia dell'arte in onore di Lionello Venturi, Rome, 1956, tome 2, p. 117-123 (p. 119 : "La solution de 1849 était la bonne. Conforme aux usages démocratiques, elle remettait aux artistes le gouvernement du Salon, la sélection des envois, la distribution des récompenses").

59. Émile Zola l'emploie encore dans son Salon de 1880 (Émile Zola, Salons, recueillis, annotés et présentés par F. W. J. Hemmings et Robert J. Niess, Genève/Paris, 1959, p. 237). 60. Ouv. cité (note 32).

61. Charles Blanc reprenait la distinction entre le grand art, qui est affaire de l'État, et l'art secondaire, destiné aux particuliers, pour lequel il préconise des associations d'acheteurs organisant des expositions avec loterie (analogues, donc, aux Kunstvereine dont la création avait commencé en Allemagne).

\section{INDEX}

Mots-clés : Arts, Administration 